\title{
Citations
}

\section{Going native}

Binding studies of membrane proteins proffer the most meaningful results when the protein conformation and context match the authentic cellular environment. Unfortunately, a prominent technology for assessing binding kinetics, surface plasmon resonance (SPR), does not facilitate analyzing proteins in their native state. In a typical SPR assay, proteins are purified and immobilized on the gold surface of the chip, which is subsequently exposed to ligand-containing solutions. The wide gulf between this and the native cell environment can be at least partially bridged by embedding the proteins in liposomes before affixing to the chip, or by depositing cell-derived membrane patches on the sensor surface. Better still to study membrane protein-ligand binding kinetics in intact, living cells. This is the goal realized by Wang et al. in an article in Nature Chemistry. In the new setup, cells are cultured on a gold-coated glass coverslip functioning as the SPR sensor. The cells are monitored by a microscope configured for simultaneous bright-field, fluorescence, and SPR imaging, while a drug perfusion system enables the solution bathing the cells to be switched in $1-2$ seconds. By following the SPR intensity over time as ligand is added and washed away, Wang et al. obtained sensorgrams, from which kinetic parameters can be derived. Since SPR depends on the interaction occurring close to the sensor surface, only binding events on the flattened edge of the cell are measured. Even so, SPR imaging has sufficient spatial resolution for local variations in binding to be distinguished within a single cell. The authors show data from three types of binding analysis: kinetic studies of glycoprotein-lectin interactions, nicotinic acetylcholine receptor distribution and activity as determined with an antibody as ligand, and tracking of

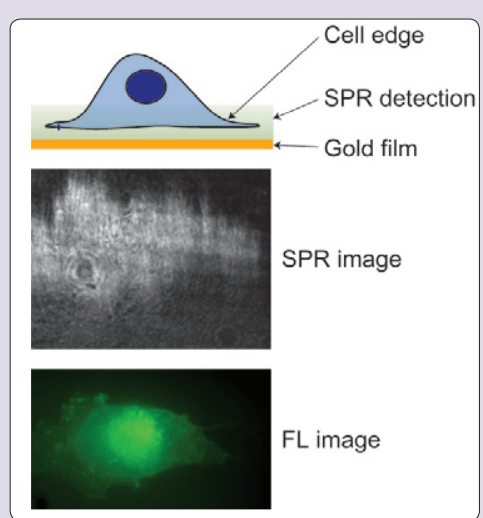

Schematic of SPR and sample image, versus fluorescence microscopy with labeled ligand. Source: Nature Chemistry glycoprotein polarization during chemotaxis (again using lectin as ligand). These examples highlight important advantages compared to standard techniques. Fluorescence and atomic force microscopy both can reveal spatial distribution, but are primarily endpoint assays and are not adept at binding kinetics. Moreover, as a label-free technique, SPR sidesteps nonspecific signal from fluorophore-conjugated secondary antibodies. A final advantage of SPR is its appropriateness for competitive binding assays, which are useful for discovering inhibitors of receptor-ligand interactions. These features should entice users looking for a noninvasive, quick-response method to understand membrane proteins in their authentic state.

Wang et al. Label-free measuring and mapping of binding kinetics of membrane proteins in single living cell. Nature Chem. [Epub ahead of print, August 26, 2012; doi:10.1038/ NCHEM.1434].

\section{Switch it up}

Those ready to replace their current photoswitchable fluorescent protein (PSFP) should seek out an article from Subach et al. in JACS. PSFPs, which are particularly useful for tracking purposes, change color when irradiated. Typically the excitation wavelength is in the $400 \mathrm{~nm}$ range, but in previous work, Subach et al. engineered PSmOrange, a PSFP that can be triggered by longer-wavelength, blue-green light. In the new publication, the authors address the relatively poor photoconversion of PSmOrange by mutagenizing it. With just six amino acid changes from the first-generation version, PSmOrange2 photoconverts more quickly and with greater overall efficiency. Although PSmOrange2 is a little less bright than its parent in the far-red (photoswitched) form, its better photoconversion translates to brighter cells. As is true for other PSFPs, PSmOrange2 is compatible with super-resolution microscopy; in addition, it can be photoswitched by standard light sources used in two-photon microscopes. The key innovation described by Subach et al. is the use of PSmOrange2 as a FRET acceptor. Since PSmOrange2 is not efficiently photoswitched by violet light, a fluorescent protein that is excited by that wavelength and whose emission spectrum overlaps with PSmOrange2's photoswitching action spectrum could act as a FRET donor to PSmOrange2. T-Sapphire fits that bill, as shown with two FRET scenarios in mammalian cells. In the first, T-Sapphire was fused to FK506 binding protein (FKBP) and PSmOrange2 to FKBP rapamycin-binding domain. In the presence of rapamycin, when the two proteins should interact, PSmOrange2 photoconversion accelerated 3.3-fold. Similarly, fusions of EGFR-T-Sapphire and PSmOrange2-Grb2 (an EGFR adaptor protein) yielded 2.3-fold faster photoconversion under EGF stimulation. Together, these data constitute the first demonstration of FRET-facilitated photoswitching. Since conversion of PSmOrange2 to its far-red form is perpetual, it is a stable indicator of a prior interaction, and offers comple- mentary information to FRET methods that detect transient binding events. This unique aspect of PSmOrange2, as well as its promising features for live cell and deepissue imaging and its compatibility with blue, cyan, and green fluorophores, should inspire many to switch to this new tool for tracking proteins and their interactions.

Subach et al. A FRET-facilitated photoswitching using an orange fluorescent protein with the fast photoconversion kinetics. J Am Chem Soc. [Epub ahead of print, August 27, 2012; doi:10.1021/ ja3034137].

Selected and written by Nijsje Dorman, Ph.D. 四

BioTechniques 53:271 (November 2012)

doi 10.2144/000113946

To purchase reprints of this article, contact: biotechniques@fosterprinting.com 